\title{
Effect of Catalyst Diameter on Vapour-Liquid-Solid Growth of GaAs Nanowires
}

B. O'Dowd, ${ }^{1}$ a) T. Wojtowicz, ${ }^{2}$ S. Rouvimov, ${ }^{3}$ X. Liu, ${ }^{4}$ R. Pimpinella, ${ }^{4}$ V. Kolkovsky, ${ }^{2}$ T. Wojciechowski, ${ }^{2}$ M. Zgirski, ${ }^{2}$ I. V. Shvets, ${ }^{1}$ M. Dobrowolska, ${ }^{4}$ J. Furdyna, ${ }^{4}$ and I.V. Shvets ${ }^{1}$

1) CRANN, School of Physics, Trinity College, the University of Dublin, Ireland

${ }^{2)}$ Institute of Physics, Polish Academy of Sciences, Warsaw, Poland

3) Notre Dame Integrated Imaging Facility (NDIIF), University of Notre Dame, IN, USA

4) Physics, University of Notre Dame, IN, USA

(Dated: 8 August 2014)

GaAs nanowires were grown on (111)B GaAs substrates using the vapour-liquid-solid (VLS) mechanism. The Au/Pt nanodots used to catalyse wire growth were defined lithographically and had varying diameter and separation. An in-depth statistical analysis of the resulting nanowires, which had a cone-like shape, was carried out. This revealed that there were two categories of nanowire present, with differing height and tapering angle.

The bimodal nature of wire shape was found to depend critically on the diameter of the Au-Ga droplet atop the nanowire. Transmission Electron Microscopy (TEM) analysis also revealed that the density of stacking faults in the wires varied considerably between the two categories of wire. It is believed that the cause of the distinction in terms of shape and crystal structure is related to the contact angle between the droplet and the solid-liquid interface. The dependency of droplet diameter on contact angle is likely related to line-tension, which is a correction to Young's equation for the contact angle of a droplet upon a surface. The fact that contact angle may influence resulting wire structure and shape has important implications for the planning of growth conditions and the preparation of wires for use in proposed devices.

a)Electronic mail: odowdbj@tcd.ie 


\section{INTRODUCTION}

The interesting structural, electronic and optical properties of free-standing III-V nanowires have generated significant interest and inspired the design of a wide variety of proposed applications $^{1-5}$. The Vapour-Liquid-Solid (VLS) mechanism is widely used to fabricate GaAs nanowires using Au nanodroplets as the catalysts for growth. Common methods for preparation of the array of $\mathrm{Au}$ droplets include annealing a continuous thin film of $\mathrm{Au}^{6,7}$ aerosol deposition of $\mathrm{Au}$ nanoparticles onto the substrate ${ }^{8}$ and drop-casting Au nanoparticles from a colloid dispersion ${ }^{9}$. While these approaches have the advantage of being able to quickly cover a large area (order $\mathrm{cm}^{2}$ ), they have the disadvantage of resulting in droplets having random location and a large distribution of diameter. These effects are known to result in a distribution in wire shape and crystal structure ${ }^{7,10-12}$. For application purposes, consistency in wire shape is likely to be very important and minimisation of the density of stacking faults is also preferable ${ }^{13-15}$. Here we present the results of GaAs nanowire growth carried out using Au nanodots which are defined using Electron-Beam Lithography (EBL). The use of EBL-defined droplets presents an opportunity for in-depth statistical analysis of wire shape and structure, as well as a means of testing the feasibility of EBL as a means of defining wire location for application purposes.

While bulk GaAs has zincblende (ZB) crystal structure, it is well known that nanostructures grown using the VLS method frequently exhibit wurtzite (WZ) structure ${ }^{7,16}$. The difference between these structures amounts to a difference in the stacking order of the (111) planes (equivalently (0001) planes in the case of WZ), with ZB having 'ABCABC' stacking while for WZ the stacking order is 'ABABAB'. The [111] direction happens to be the most common growth direction for VLS-grown GaAs nanowires, and so the wires grow by nucleating (111)/(0001) planes at the solid-liquid interface.

While the the lower surface energy of the facets of WZ GaAs has a role to play in the preference for this crystal structure in nanostructures, this is not the primary cause, as was revealed by Glas et al. ${ }^{16}$. They examined the energy cost of adding a single (111) layer at the solid-liquid interface, and showed that in the case of ZB structure, there is a dependency on the angle made by the droplet edge and the plane of the solid-liquid interface, known as the contact angle ' $\theta_{c a}$ '. This is due to the fact that the sidewalls of a nanowire having ZB crystal structure are $\{111\}$ planes which make an angle of $19.5^{\circ}$ with the wire axis, whereas for WZ 
structure the sidewall facets (either $\{11 \overline{2} 0\}$ or $\{10 \overline{1} 0\}$ ) are exactly parallel to the [111] wire axis. Thus, the contact angle of the droplet can affect the preference for one crystal structure over another, with $\theta_{c a}$ close to $90^{\circ}$ giving preference to $\mathrm{WZ}$ crystal structure, while a contact angle significantly greater or less than $90^{\circ}$ will be more likely to give rise to the nucleation of ZB layers.

The contact angle for a droplet on a surface is given by Young's equation ${ }^{17,18}$ :

$$
\gamma_{L V} \cos \theta_{c a}=\gamma_{S V}-\gamma_{S L}
$$

where $\gamma$ is the interfacial energy, and the suffixes S, L and V denote that the interface is between solid, liquid and vapour respectively. Two modifications can be made to this equation to more accurately represent the circumstances for a droplet on top of a nanowire. Firstly an effect known as line tension, which is "the excess free energy per unit length of a contact line where three distinct phases coexist" ${ }^{19}$ becomes important on the nanoscale. The line tension, denoted $\tau$, acts along the solid-liquid interface and can be directed inwards or outwards on the circumference of the droplet base, thereby altering the contact angle. It varies as $1 / r$, where $r$ is the radius of the solid-liquid contact area ${ }^{17,18,20,21}$. The second modification takes into account the fact that the droplet is essentially pinned by the boundaries of the solid-liquid interface. This term depends on the inclination angle, $\alpha$, between the wire sidewalls and the plane of the solid-liquid interface ${ }^{22,23}$. Including these terms gives us the modified Young's equation:

$$
\gamma_{L V} \cos \theta_{c a}=\gamma_{S V} \cos \alpha-\gamma_{S L}-\frac{\tau}{r}
$$

The introduction of the line tension term introduces a size dependency of contact angle on the droplet volume. If $\tau$ is positive then the contact angle is increased for very small droplets. However, except for values close to zero, a positive value of $\tau$ will result in a termination

of growth at a certain height, and so for steady state growth $\tau$ is usually assumed to be negative ${ }^{17,19,22,24}$.

\section{METHOD}

Ordered arrays of Au-Pd nanodots (85\% Au, 15\% Pd) having thickness of $20 \mathrm{~nm}$ were fabricated on a GaAs (111)B substrate using EBL. The Pd is included to ensure adhesion 
of the nanodots to the GaAs substrate. The nanodots are arranged in square grids, with 3 values of diameter $(30,60$ and $100 \mathrm{~nm})$ and four values of centre-to-centre separation (0.5, 1.0, 1.5 and $2.0 \mu \mathrm{m}$ ) giving a total of 12 grids (each grid measuring $100 \mu \mathrm{m} \times 100 \mu \mathrm{m}$ ). The patterned sample is loaded into a Riber $32 \mathrm{MBE}$ system under ultra-high vacuum alongside an unpatterned GaAs (111)B sample having a continuous $10 \AA$-thick film of Au. Both samples are first subjected to a deoxidation anneal at $600^{\circ} \mathrm{C}$, which also causes the thin $\mathrm{Au}$ film of the unpatterned sample to form a random array of Au-Ga droplets. This unpatterned sample represents the 'conventional' method of nanowire growth via VLS. The unpatterned sample also provides wires for TEM analysis.

During growth, Ga is evaporated onto the substrate by means of a standard Knudsen cell, while $\mathrm{As}_{2}$ molecules are generated via an arsenic-cracker source, with cracker-zone heated to $1000^{\circ} \mathrm{C}$. The substrate temperature is $480^{\circ} \mathrm{C}$ during growth and the substrate stage is rotated about its normal axis. Growth is carried out in As-rich conditions (V/III ratio is approximately 15). Following growth, nanowires from each of the 12 arrays are examined using SEM (FEI Magellan 400 FESEM) and the crystal structure of wires from the unpatterned sample are analysed using HRTEM (FEI Titan 80-300).

\section{RESULTS AND DISCUSSION}

Figure 1(a) shows an individual nanowire from an array. It has a hexagonal pyramid shape, with a taper ratio (height/base radius) of approximately 25. Figure 1(b) shows a portion of one of the nanowire arrays grown from a grid-array of $\mathrm{Au}_{0.85} \mathrm{Pd}_{0.15}$ nanodots. In this case the nanodots had a separation of $1 \mu \mathrm{m}$. The rows of wires in the array are visible but are slightly distorted by missing wires or the existence of two wires at a single site, which may be the result of patterned nanodots splitting into a number of smaller droplets during the deoxidation anneal. A closer view of the facet structure is shown in figure 1(c). In this image the $[11 \overline{2}]$ direction is to the right, meaning that the facets that make up the wire sidewalls (which are highlighted in red) are $\{11 \overline{2}\}$ if the crystal structure is ZB, or $\{10 \overline{1} 0\}$ if it is WZ. The appearance of wires possessing a rod-like shape with uniform diameter equal to that of the catalysing droplet is usually considered to be indicative of a procedure whereby Ga adatoms landing on the wire sidewalls migrate to the solid-liquid interface where they incorporate into the wire ${ }^{7,9,25}$. The observation of cone-shaped wires in the present case is 


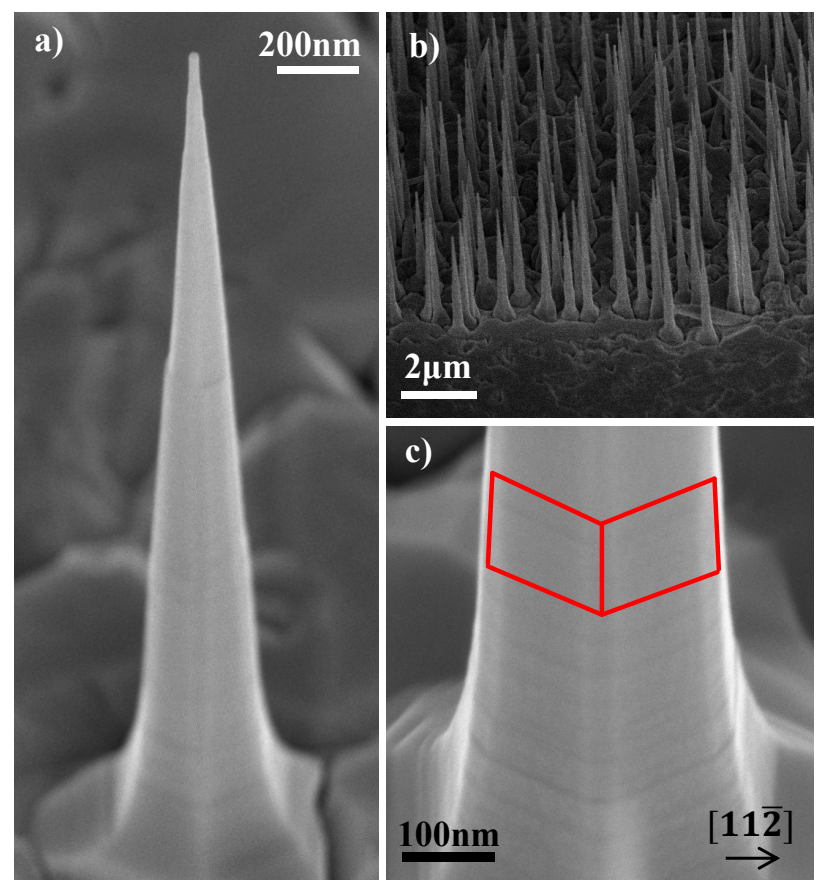

FIG. 1. SEM images of nanowires grown via VLS mechanism from EBL defined nanodots. A close-up of one wire is shown in (a), and a portion of one of the patterned regions is shown in (b). In (c), which is a close-up of the base of the wire shown in (a), the facet structure of the wire can be seen.

likely due to the ability of a portion of the Ga adatoms to incorporate at the wire sidewalls leading to radial growth. This may in turn be due to lower diffusion length on the sidewalls or higher reactivity of the adatoms with the As dimers in these growth conditions. A study by Sartel et al. found greater rates of radial growth when $\mathrm{As}_{2}$ was used, as in the case of the present study, rather than $\mathrm{As}_{4}{ }^{26}$.

A detailed examination was carried out measuring the height, tapering angle and the diameter of the droplet atop the wires, $\varphi$, for nanowires in each of the 12 patterned arrays of the sample. A total of 987 wires were measured in this way. A 2D density plot was produced which measured the number of wires falling within particular values for height and angle ('bin' sizes for height and angle are $250 \mathrm{~nm}$ and $0.5^{\circ}$ respectively). The results are shown in figure 2(a). As can be seen, the nanowires seem to be grouped into one of two regions. One, towards the top left of the plot (highlighted by a black dashed circle) is centred around a nanowire height of $4.2 \mu \mathrm{m}$ and a taper angle of $4^{\circ}$. Wires from this group shall be referred to as category 1 wires. The other wire group, towards the bottom right (highlighted by a white dashed circle) is centred around a height of $3 \mu \mathrm{m}$ and an angle of approximately $6.5^{\circ}$. 
FIG. 2. (a) 2D density plots of height and angle for all 987 wires measured, with wires conforming to category 1 and category 2 characteristics highlighted by black and white dashed circles, respectively. 2D density plots for subsets of wires with droplet diameters in the regions (b) 60-80nm, (c) 40-60nm, (d) 30-40nm, (e) 20-30nm and (f) 0-20nm.

Wires from this group shall be referred to as category 2 wires.

Following further analysis, it was discovered that the likelihood of a wire belonging to a particular group was dependent on the droplet diameter $\varphi$. To illustrate this, the density plot of all 987 wires shown in figure 2(a) was broken down into subsets based on the diameter of the droplet. The subsets chosen are 60-80nm, 40-60nm, 30-40nm, 20-30nm and 0-20nm, and the 2D density plots for these subsets are shown in figures 2(b)-(f). As can be seen, for large values of $\varphi$ the wire is most likely to belong to category 1 (i.e. height and taper angle of approximately $4 \mu \mathrm{m}$ and $4^{\circ}$ respectively). At around $\varphi=30 \mathrm{~nm}$ there is a transition, below which the wires are more likely to belong to category 2 (i.e. height of $3 \mu \mathrm{m}$ and taper angle of $\left.6.5^{\circ}\right)$. It is interesting that the transition is not a gradual shift from one category to the other as $\varphi$ is reduced but rather a shift in the population densities of the two categories. This suggests that there are two distinct preferred shapes of wire depending on the droplet diameter $\varphi$.

To investigate the origin of the dependency of wire shape on droplet diameter, wires from the unpatterned sample were examined using Transmission Electron Microscopy (TEM). Figure 3(a) shows an image taken near the top of a category 1 wire which has a droplet diameter of $60 \mathrm{~nm}$. The Au-Ga droplet appears as a dark, truncated ellipsoid at the top of the wire. A large number of stacking faults appear as bands of lines crossing the growth axis of the wire. A high-resolution image of a typical region of this wire is shown in $3(\mathrm{~b})$. In this picture, a coloured line indicates the crystal structure of that portion of the wire, with red representing WZ structure, and blue and green representing the two possible orientations of ZB growth when viewed along the $[1 \overline{1} 0]$ direction. The average spacing between stacking faults is between 1-2nm. The edges of the wire are not perfectly straight, and it is noticed that wire diameter does not decrease continuously as a function of distance from the foot of the wire. An example of this can be seen at the bottom of figure 3(a).

Figure 3(c) shows a TEM image taken from a wire from the unpatterned sample having a droplet diameter of $17.5 \mathrm{~nm}$ (a category 2 wire according to the criteria above). The Au-Ga 


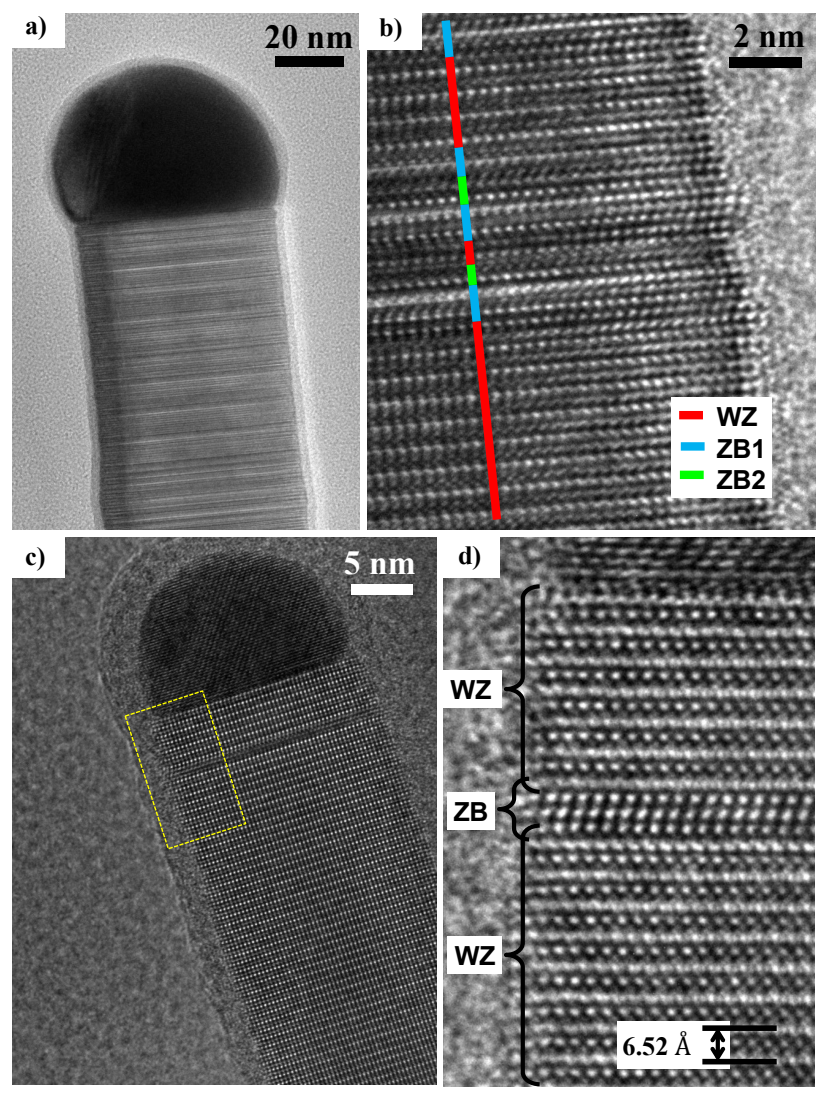

FIG. 3. (a) TEM image of a category 1 wire with droplet diameter of $60 \mathrm{~nm}$. (b) High resolution image of typical region from (a). Coloured lines represent the different crystal structures of along the wire length. (c) TEM image of a category 2 wire with droplet diameter of $17.5 \mathrm{~nm}$. (d) HRTEM image of area highlighted by yellow rectangle in (c). 'WZ' and 'ZB' denote wurtzite and zincblende crystal structure respectively.

droplet appears as a dark hemisphere, and it is clear that the density of stacking faults is far lower than that seen for the category 1 wire shown in 3(a). A closer view of the area highlighted by the yellow rectangle in 3(c) is shown in 3(d). Here we can see that a single atomic layer stacking fault has given rise to a zincblende layer ('ZB' in figure 3(d)) while the remainder is wurtzite ('WZ' in $3(\mathrm{~d})$ ). The average separation of stacking faults is greater than $8 \mathrm{~nm}$, and the vast majority of stacking faults consisted of single atomic layer defects as shown in figure 3(d). For the category 1 nanowire, however, the average separation between defects was 1-2nm, and ZB defects that extended for a number of atomic layers were common.

As described in the introduction section, WZ crystal structure is expected for VLS growth of GaAs nanowires where the contact angle is close to $90^{\circ}$, otherwise $\mathrm{ZB}$ defects may be 


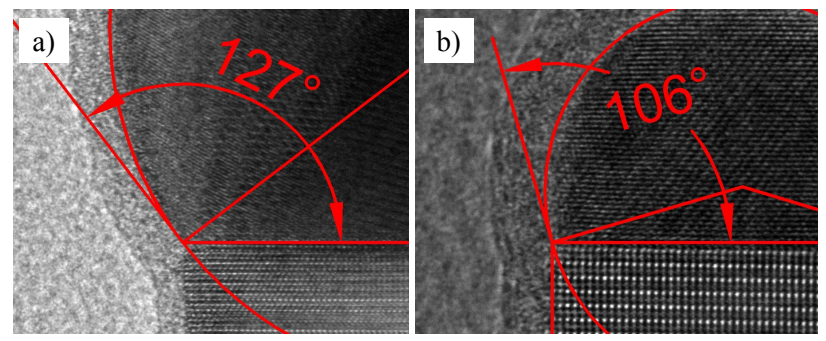

FIG. 4. TEM images showing the contact angle for category 1 (a) and category 2 (b) nanowires. expected. TEM images of the contact angles of the two wires in figure 3 are shown in figure 4. As can be seen, the contact angle for the category 1 nanowire (which has a droplet diameter of $60 \mathrm{~nm}$ ) is approximately $127^{\circ}$, whereas for the category 2 nanowire the value is only $106^{\circ}$. It appears that the larger contact angle belonging to category 1 wires has given rise to the high density of $\mathrm{ZB}$ defects observed, whereas the contact angle of the category 2 wire, being closer to $90^{\circ}$, has allowed WZ growth with a much lower density of ZB defects. The bimodal nature of the shapes of the wires expressed in figure 2 is likely to also be related to the differing contact angle for category 1 and 2 wires. For category 1 wires the diameter of the droplet is significantly greater than that of the wire. Therefore, it is possible that during the growth a new GaAs layer will nucleate at a point slightly beyond the width of the wire itself, resulting in an increase in wire diameter (reverse tapering). This is seen on occasion in the TEM analysis, as evident towards the bottom of figure 3(a). Hence the tapering angle of category 1 wires is lower than that of category 2 . The difference in height between the two categories of wires may be related to the fact that there is little variation in volume from one category to the other (less than $3 \%$ difference in average volume of wires with $\varphi=0-30 \mathrm{~nm}$ and $\varphi=40-80 \mathrm{~nm})$. If volume is constant for both categories of wire then the height must be greater in category 2 to compensate for the lower tapering angle. The similarity in volume for both categories of wire suggests that the growth rate is not sensitive to wire shape, but is governed by the quantity of material incident on the substrate per unit area.

The origin for the dependency of contact angle on droplet diameter is uncertain. Line tension, as outlined in the introduction, is one potential cause for this relationship. As described above, it is known to affect nanodroplets and varies inversely with the radius of the solid-liquid interface, $r$. From equation 2, the finding that contact angle is reduced for smaller droplets indicates that $\tau<0$, in agreement with previous findings ${ }^{17,19,22,24}$. 
It is possible that some cause other than line tension is responsible for the relationship between droplet diameter and contact angle. It may be the case that the Au:Ga ratio within the droplet varies slightly as a function of droplet diameter, or that this ratio is heterogeneous across the sample surface in a manner that depends on $\varphi$. Both of these effects are known to influence contact angle while not directly linked to line tension ${ }^{27}$. Another potential mechanism whereby droplet diameter may influence crystal structure is related to the location within the droplet at which nucleation of a new layer occurs. It is known that if nucleation occurs not at the triple-phase line, but at some point of the solid-liquid interface away from the droplet perimeter, then ZB structure is preferred ${ }^{16}$. Thus, for larger droplets, where the solid-liquid interface has a greater surface to perimeter ratio, there may be an increased likelihood of nucleation away from the triple-phase line resulting in nucleation of ZB layers. If this is the cause, then rather than the contact angle determining the crystal structure (as is assumed for the other potential causes), it may be the case that it is the crystal structure which determines the contact angle, due to the inclination angle of the $\{111\}$ wire sidewalls which are associated with ZB growth.

In summary, the size of the catalysing droplet affects the wire shape via a two-step process. Firstly, the size of the droplet influences the contact angle. Line tension has been identified as a potential mechanism for this adjustment. If line tension is indeed the cause, then our finding of larger contact angles for larger droplets indicates a negative value of $\tau$, which is in agreement with separate studies ${ }^{17,19,22,24}$. Secondly, the contact angle influences both the crystal structure and the angle of tapering, the former due to the finding by Glas et al. that WZ and ZB nucleation energies are contact angle dependent ${ }^{16}$, and the latter due to the potential for layers to nucleate beyond the boundary of the underlying layer, as observed experimentally (bottom of figure $3(\mathrm{a})$ ).

Other potential mechanisms whereby droplet diameter may bring about the findings observed involve the composition of the AuGa droplet and the potential for nucleation to occur away from the triple-phase line more readily in larger droplets ${ }^{16,27}$. In the latter case, it is possible that the crystal structure determines the contact angle rather than the other way around.

However, the ability of contact angle to dictate crystal structure seems to be borne out from an analysis of previous studies. For example, there are a number of investigations where the diameter of the droplet is considerably larger than the solid-liquid interface (many of 
these are self-catalysed wires), and the structure is found to be predominantly $\mathrm{ZB}^{28-33}$. In contrast, most studies which find predominantly WZ structure find a contact angle close to $90^{\circ}$ (in cases where contact angle was shown or reported) $)^{7,12,34,35}$. In addition, a number of studies also found a greater density of ZB defects/regions near the foot of nanowires ${ }^{12,16,36,37}$. This is likely due to the fact that during the initial stages of nanowire growth the contact angle is much less than $90^{\circ}$, since the catalysing droplet is situated on a flat surface and the angle $\alpha$ in equation 2 is therefore equal to zero.

\section{CONCLUSIONS}

GaAs nanowires were successfully grown from Au catalyst nanodots which were defined lithographically. The pattern of the nanowire array broadly reflects that of the grid of $\mathrm{Au}$ nanodots, with a small proportion of deviations due to missing wires and multiple wires per nanodot site. The positioning of wires in this way allows a thorough statistical analysis.

It was found that the droplet diameter has a critical influence on the resulting shape of the nanowires, with two distinct categories emerging in terms of height and tapering angle. These categories were not apparent from a cursory visual inspection of the SEM images. It is believed that the mechanism by which droplet diameter influences wire shape is via the dependency of line tension on droplet radius. Line tension determines the contact angle of the droplet, which in turn affects the crystal structure and wire shape. Aside from line tension, other mechanisms which may be responsible for the relationship between droplet size and crystal structure are also explored. An examination of the literature suggests that there is a strong correspondence between the contact angle and the specific crystal structure, and yet this relationship is very rarely discussed. These findings highlight the importance of considering droplet shape when reporting results relating to nanowire growth and when planning growth conditions, particularly when a specific crystal structure or minimisation of defects is central to the functionality of proposed devices.

\section{ACKNOWLEDGMENTS}

Research in Ireland is supported by the Science Foundation Ireland (SFI) under Contract No. 06/IN.1/I91, the Irish Government's Program (ISPIRE) for Research in Third Level 
Institutions, Cycle 4, National Development Plan 2007-2013 and the Naughton Fellowship Program. The work at Notre Dame was supported by the National Science Foundation grant DMR1005851. The research in Poland was partially supported by the European Union within European Regional Development Fund, through grant Innovative Economy (POIG.01.01.02-00-008/08).

\section{REFERENCES}

${ }^{1}$ D. P. Burt, N. R. Wilson, J. M. R. Weaver, P. S. Dobson, and J. V. Macpherson, Nano Letters 5, 639 (2005).

${ }^{2}$ G. Mariani, A. C. Scofield, C. H. Hung, and D. L. Huffaker, Nature Communications 4, 1497 (2013).

${ }^{3}$ J. Chen, Y. Y. Dai, J. Luo, Z. L. Li, S. Z. Deng, J. C. She, and N. S. Xu, Applied Physics Letters 90, 253105 (2007).

${ }^{4}$ M. S. Gudiksen, L. J. Lauhon, J. Wang, D. C. Smith, and C. M. Lieber, Nature 415, 617 (2002).

${ }^{5}$ K. A. Dick, Progress in Crystal Growth and Characterization of Materials 54, 138 (2008).

${ }^{6}$ K. Tivakornsasithorn, R. E. Pimpinella, V. Nguyen, X. Liu, M. Dobrowolska, and J. K. Furdyna, Journal of Vacuum Science \& Technology B: Microelectronics and Nanometer Structures 30, 02B115 (2012).

${ }^{7}$ H. Shtrikman, R. Popovitz-Biro, A. Kretinin, L. Houben, M. Heiblum, M. Bukala, M. Galicka, R. Buczko, and P. Kacman, Nano Letters 9, 1506 (2009).

${ }^{8}$ M. H. Magnusson, K. Deppert, J. O. Malm, J. O. Bovin, and L. Samuelson, Nanostructured Materials 12, 45 (1999).

${ }^{9}$ P. Paiano, P. Prete, N. Lovergine, and A. M. Mancini, Journal of Applied Physics 100, 094305 (2006).

${ }^{10}$ J. C. Harmand, G. Patriarche, N. Pere-Laperne, M.-N. Merat-Combes, L. Travers, and F. Glas, Applied Physics Letters 87, 203101 (2005).

${ }^{11}$ M. Plante and R. LaPierre, Journal of Crystal Growth 286, 394 (2006).

${ }^{12}$ M. Plante and R. LaPierre, Journal of Crystal Growth 310, 356 (2008).

${ }^{13}$ S. Q. Wang and H. Q. Ye, Journal of Physics: Condensed Matter 14, 9579 (2002). 
${ }^{14}$ K. A. Dick, P. Caroff, J. Bolinsson, M. E. Messing, J. Johansson, K. Deppert, L. R. Wallenberg, and L. Samuelson, Semiconductor Science and Technology 25, 024009 (2010).

${ }^{15}$ J. Johansson, L. S. Karlsson, C. P. T. Svensson, T. Martensson, B. a. Wacaser, K. Deppert, L. Samuelson, and W. Seifert, Nature Materials 5, 574 (2006).

${ }^{16}$ F. Glas, J.-C. Harmand, and G. Patriarche, Physical Review Letters 99, 3 (2007).

${ }^{17}$ H. K. Guo and H. P. Fang, Chinese Physics Letters 22, 787 (2005).

${ }^{18}$ L. Schimmele and S. Dietrich, The European Physical Journal B 30, 427 (2009).

${ }^{19}$ J. H. Weijs, A. Marchand, B. Andreotti, D. Lohse, and J. H. Snoeijer, Physics of Fluids 23, 022001 (2011).

${ }^{20}$ B. Pethica, Journal of Colloid and Interface Science 62, 567 (1977).

${ }^{21}$ P. Chen, J. Gaydos, and A. W. Neumann, Langmuir 12, 5956 (1996).

${ }^{22}$ V. Schmidt, S. Senz, and U. Gösele, Applied Physics A 80, 445 (2004).

${ }^{23}$ N. Li, T. Tan, and U. Gösele, Applied Physics A 86, 433 (2007).

${ }^{24}$ J. Bauer, V. Gottschalch, H. Paetzelt, G. Wagner, B. Fuhrmann, and H. Leipner, Journal of Crystal Growth 298, 625 (2007).

${ }^{25}$ V. Pankoke, S. Sakong, and P. Kratzer, Physical Review B 86, 085425 (2012).

${ }^{26}$ C. Sartel, D. Dheeraj, F. Jabeen, and J. Harmand, Journal of Crystal Growth 312, 2073 (2010).

${ }^{27}$ A. Amirfazli and A. W. Neumann, Advances in colloid and interface science 110, 121 (2004).

${ }^{28}$ Y. Heon Kim, D. Woo Park, and S. Jun Lee, Applied Physics Letters 100, 033117 (2012).

${ }^{29}$ T. Rieger, S. Heiderich, S. Lenk, M. I. Lepsa, and D. Grützmacher, Journal of Crystal Growth 353, 39 (2012).

${ }^{30}$ G. E. Cirlin, V. G. Dubrovskii, Y. B. Samsonenko, A. D. Bouravleuv, K. Durose, Y. Y. Proskuryakov, B. Mendes, L. Bowen, M. A. Kaliteevski, R. A. Abram, and D. Zeze, Physical Review B 82, 035302 (2010).

${ }^{31}$ B. Bauer, A. Rudolph, M. Soda, A. Fontcuberta i Morral, J. Zweck, D. Schuh, and E. Reiger, Nanotechnology 21, 435601 (2010).

${ }^{32}$ S. Gibson and R. LaPierre, Physica Status Solidi (RRL) - Rapid Research Letters 7, 845 (2013).

${ }^{33}$ S. Plissard, K. A. Dick, G. Larrieu, S. Godey, A. Addad, X. Wallart, and P. Caroff, Nanotechnology 21, 385602 (2010). 
${ }^{34}$ D. L. Dheeraj, G. Patriarche, H. Zhou, T. B. Hoang, A. F. Moses, S. Gronsberg, A. T. J. van Helvoort, B. O. Fimland, and H. Weman, Nano Letters 8, 4459 (2008).

${ }^{35}$ M. Tchernycheva, J. C. Harmand, G. Patriarche, L. Travers, and G. E. Cirlin, Nanotechnology 17, 4025 (2006).

${ }^{36}$ J. Bauer, H. Paetzelt, V. Gottschalch, and G. Wagner, Physica Status Solidi (B) 247, 1294 (2010).

${ }^{37}$ F. Glas, G. Patriarche, and J. C. Harmand, Journal of Physics: Conference Series 209, $012002(2010)$. 